\title{
NOTAS
}

\section{Evaluación del muestreo por recuento angular de Bitterlich en distintas distribuciones espaciales y diamétricas generadas por simulación}

\author{
Evaluation of Bitterlich angular count sampling in different spatial patterns and diametric \\ distributions by the means of simulations
}

\author{
Marcelo Arturi \\ Universidad Nacional de La Plata, Facultad de Ciencias Agrarias y Forestales, Laboratorio de Investigación de Sistemas Ecológicos \\ y Ambientales, diagonal $113 \mathrm{~N}^{\circ}$ 469, La Plata, Argentina, tel.: +542214271442, marceloarturif@gmail.com
}

\begin{abstract}
SUMMARY
The precision and accuracy of Bitterlich angular count (BAC) for the estimation of basal area density could depend on diametric distribution since the inclusion probability of the trees increases with their basal area. Trees spatial pattern can also influences since point-tree distances determine their inclusion in the sample. In this study, the precision and bias of BAC under different diametric distribution and spatial patterns were evaluated by the means of simulations. Normal and lognormal diametric distributions were simulated with basal area density ranging between $30-33 \mathrm{~m}^{2} \mathrm{ha}^{-1}$. These diametric distributions were simulated in three spatial patterns: regular, random and clumped yielding six combinations. A seventh class was simulated with bimodal diametric distribution and a mixed spatial pattern: clumped for small trees and random for large trees. Bias was less than $0.10 \mathrm{~m}^{2} \mathrm{ha}^{-1}$ except for clumped pattern with lognormal diametric distribution and mixed pattern. In these cases bias tended to be negative $\left(-0.15 \mathrm{a}-0.25 \mathrm{~m}^{2}\right.$ ha $\left.{ }^{-1}\right)$. The lowest mean absolute deviation (MAD) was observed for the regular pattern 3-7 \%. In the clumped pattern it ranged 7-10\% and 8-11 \% in the mixed. MAD tended to be slightly lower in the normal distribution than in lognormal in the regular spatial pattern but the reverse trend was observed in the clumped pattern. In practical terms BAC yields null bias and low MAD with a low intensity sampling for a wide variety of stand structures.
\end{abstract}

Key words: bias, basal area, DBH, forest structure, forest biometry.

\section{RESUMEN}

La precisión y exactitud del recuento angular de Bitterlich para estimar la densidad en área basal podrían depender de la distribución diamétrica, porque la probabilidad de inclusión de los árboles aumenta con su área basal. La distribución espacial de los árboles también podría influir, ya que de ella dependen las relaciones de distancia punto-árbol que determinan su inclusión en la muestra. En este trabajo se evaluó la precisión y el sesgo del recuento angular de Bitterlich en diferentes distribuciones diamétricas y espaciales mediante simulaciones. Se simularon distribuciones diamétricas lognormales y normales con densidades de $30-33 \mathrm{~m}^{2}$ ha-1 y se establecieron distribuciones espaciales regular aleatoria y agrupada, resultando de ello seis combinaciones estructurales. Una séptima situación se presentó como una distribución bimodal con árboles de mayor diámetro distribuidos aleatoriamente y los menores agrupados (distribución espacial mixta). El sesgo no se diferenció de $0 \mathrm{y}$ fue $<0,10 \mathrm{~m}^{2} \mathrm{ha}^{-1}$ En la distribución espacial agrupada con distribución diamétrica lognormal y en la distribución espacial mixta tendió a ser negativo $\left(-0,15\right.$ a $0,25 \mathrm{~m}^{2}$ ha $\left.{ }^{-1}\right)$. El desvío absoluto medio presentó sus valores más bajos en la distribución regular (3-7 \%) y mayores en las distribuciones agrupada (7-10 \%) y mixta (8-11\%). Esos desvíos tendieron a ser mayores en la distribución lognormal que en la normal para patrones regulares, pero se observó una tendencia opuesta en el patrón agrupado y mixto. En términos prácticos el recuento angular fue eficiente e insesgado en un amplio rango de distribuciones espaciales y diamétricas.

Palabras clave: sesgo, área basal, DAP, estructura forestal, biometría forestal.

\section{INTRODUCCIÓN}

La estimación de la densidad en área basal mediante recuento angular fue propuesta por Bitterlich (1984) y se la conoce también como muestreo de Bitterlich. Consiste en la proyección de un ángulo fijo y el conteo de los árboles cuya sección a $1,30 \mathrm{~m}$ de altura proyecta un ángulo ma- yor que el establecido previo al muestreo. Se lo considera un procedimiento práctico, eficiente e insesgado (Whyte y Tennent 1975). Incluso, en aplicaciones del muestreo angular para estimar diámetros y volúmenes ha probado ser eficiente e insesgado (Salas et al. 2005). Sus propiedades son conocidas por los resultados de su aplicación en una amplia variedad de situaciones prácticas (Gregoire y Va- 
lentine 2007, Eastaugh y Hasenauer 2014) y por aproximaciones teóricas (Palley y Horwitz 1961, Sukwong et al. 1971, Eastaugh y Hasenauer 2014). Dado que la probabilidad de que un árbol sea incluido es proporcional a su área basal, este procedimiento presenta una mayor intensidad de muestreo para los árboles de mayor tamaño que para los más pequeños (Kangas y Maltamo 2006, Gregoire y Valentine 2007). Si bien los árboles de menor diámetro a 1,30 $\mathrm{m}$ de altura (DAP) son los que aportan menor área basal, aún cuando sean importantes numéricamente, la diferencia en las probabilidades de inclusión podrían determinar que la precisión del muestreo y tal vez su propiedad de insesgado, dependan de la distribución diamétrica del bosque muestreado. Además, es esperable que la incidencia de la distribución diamétrica en estimación del área basal por recuento angular, dependa del patrón de distribución espacial de los árboles. La inclusión de los árboles en el muestreo depende de su diámetro, del ángulo de conteo y su distancia al punto de conteo. Es un hecho conocido que en los métodos para determinar la densidad, basados en mediciones de las distancias entre los puntos de muestreo y los árboles, tienden a producir estimaciones sesgadas cuando el patrón de distribución espacial de los árboles no es aleatorio (Batcheler 1971, Pollard 1971, Engeman et al. 1994, Hijbeek et al. 2013). Si en el muestreo por recuento angular, el ángulo proyectado es amplio (mayor factor de área basal del instrumento) los árboles pequeños sólo se incluyen si resultan muy cercanos al punto de muestreo. En consecuencia, en cada punto, se estima el área basal del entorno muy cercano de la estación de conteo para los árboles pequeños y de un entorno mayor para los árboles mayores. Esta diferencia en la escala espacial del muestreo para árboles de diferentes tamaños plantea la necesidad de conocer cómo se ven afectadas las estimaciones ante diferentes distribuciones diamétricas y diferentes patrones de distribución espacial de los árboles. La distribución espacial regular, con una distribución diamétrica normal, representaría la situación más homogénea mientras que una distribución espacial agregada con una distribución diamétrica irregular (de "j" invertida") representaría una situación de alta variabilidad. Especialmente compleja podría ser la situación en la que los árboles de mayor tamaño presenten una distribución espacial aleatoria o regular y los menores lo estén en una agrupada, como fue descrito en para diferentes bosques (Li et al. 2009, Burns et al. 2010).

La posible existencia de sesgo en las estimaciones por recuento angular fueron evaluadas en relación con problemas de visibilidad (Ritter et al. 2013). Los análisis de las propiedades del recuento angular de Bitterlich (Palley y Horwitz 1961, Sukwong et al. 1971, Eastaugh y Hasenauer 2014) no han abordado específicamente la incidencia de las variaciones conjuntas del patrón de distribución espacial y la distribución diamétrica. En el presente trabajo se postula que la precisión y el sesgo de la estimación por recuento angular podrían depender del patrón de distribución espacial de los árboles, la distribución diamétrica y el ángulo o factor utilizado. A fin de explorar dicha hipótesis se analiza la precisión y el sesgo de la estimación del área basal mediante simulaciones con diferentes patrones de distribución espacial, distribución diamétrica y factores de área basal.

\section{MÉTODOS}

Se realizaron simulaciones en las cuales se combinaron tres patrones de distribución espacial: regular, aleatorio y agrupado (figura 1) y dos distribuciones diamétricas: normal y lognormal (figura 2). Además de estas seis combinaciones se simuló una séptima con una distribución diamétrica bimodal y una distribición espacial agrupada para los árboles menores de $30 \mathrm{~cm}$ de DAP y aleatoria para mayores de $30 \mathrm{~cm}$ de DAP. Todas las simulaciones se realizaron en el programa estadístico de uso libre $\mathrm{R}$ ( $\mathrm{R}$ Core Team 2012) y representaron 16 ha de bosque $(400 \times 400 \mathrm{~m})$ con una densidad de aproximadamente 650 árboles ha ${ }^{-1} \mathrm{y}$ entre 30 y $33 \mathrm{~m}^{2} \mathrm{ha}^{-1}$.

- Distribución espacial regular (REG): se establecieron 10.400 árboles (650 árboles ha ${ }^{-1} \times 16$ ha) mediante una grilla cuadrangular de aproximadamente $3,9 \mathrm{~m}$ de lado. Luego se agregó una distorsión aleatoria a la posición de cada árbol entre 0 y $1 \mathrm{~m}$ con la finalidad de transformarla en una situación más asimilable a un bosque regular.

- Distribución espacial aleatoria (AZA): se establecieron 10.400 árboles cuyas coordenadas en metros fueron establecidas mediante una función aleatoria con probabilidad uniforme: runif( ).

- Distribución espacial agrupada (AGR): se utilizó la función rMatClust del paquete "spatstat" (Baddeley y Turner 2005). Esta función determina una distribución agrupada mediante la definición de tres parámetros: la densidad promedio de grupos, su radio promedio y el número promedio de árboles por grupo. Los valores utilizados fueron 0,016 (grupos $\mathrm{m}^{-2}$ ), siete metros y cuatro árboles, respectivamente. Estos valores generaron densidades de árboles que variaron entre $575 \mathrm{y}$ 700 árboles $\mathrm{ha}^{-1} \mathrm{y}$ así resultaron comparables con las densidades utilizadas para las distribuciones espaciales regular y aleatoria. El tamaño de los grupos se decidió sobre la base de los resultados encontrados en bosques de diferentes regiones del mundo que indican frecuentemente patrones de agrupamiento a una escala espacial entre 5 y $10 \mathrm{~m}$ (Condit et al. 2000, Getzin et al. 2006, Burns et al. 2010).

- Distribución espacial mixta (MIX): se asignó una distribución agrupada a los árboles de menor diámetro y una aleatoria para los de mayor diámetros generados en la distribución diamétrica bimodal.

Distribuciones diamétricas. Se utilizó la distribución normal y lognormal para definir distribuciones diamétricas regulares e irregulares respectivamente, debido a que re- 

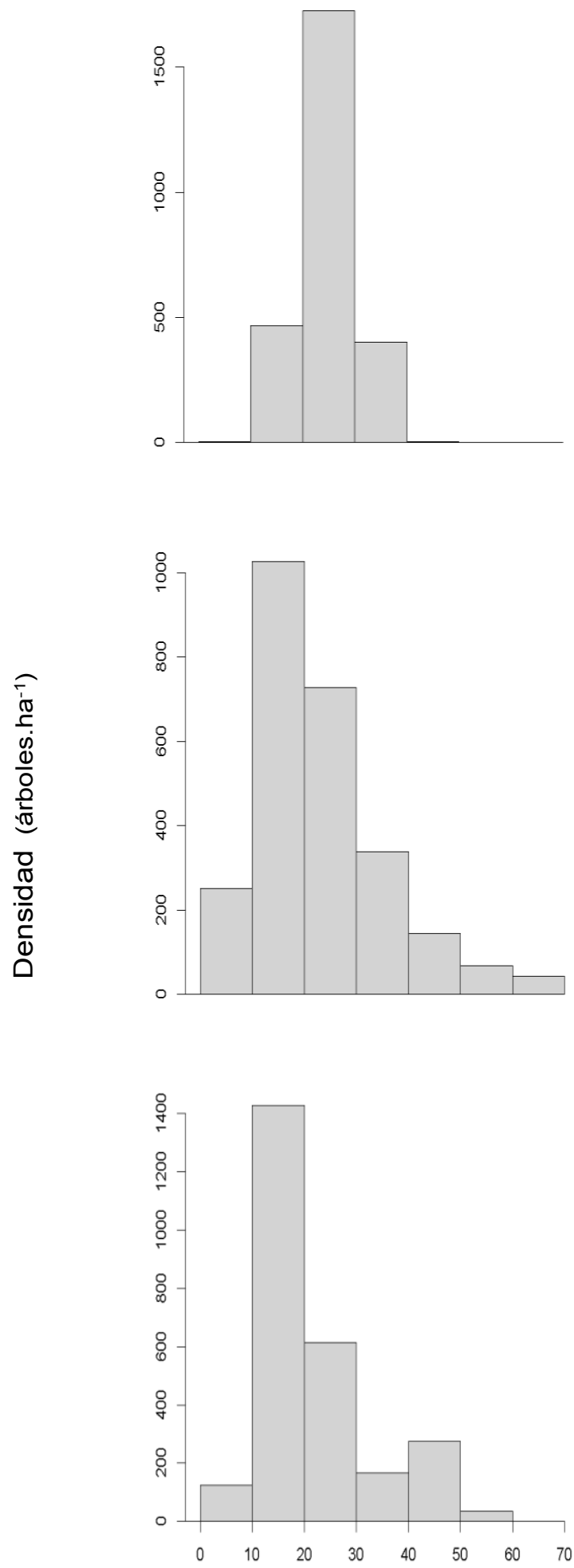

\section{$\operatorname{DAP}(\mathrm{cm})$}

Figura 1. Distribuciones diamétricas simuladas: normal (arriba), lognormal (centro) y biomodal (abajo).

Simulated diametric distributions: normal (above), lognormal (center) and bimodal (below). presentan modelos de distribución de probabilidades frecuentemente utilizados en esos casos (Podlaski y Zasada 2008, Sheykholeslami et al. 2011).

- Normal (NOR). Se asignaron diámetros aleatorios con distribución normal mediante la función $\operatorname{rnorm}()$ con una media de $25 \mathrm{~cm}$ y un desvío estándar de $5 \mathrm{~cm}$. La distribución fue truncada en $5 \mathrm{~cm}$ como límite inferior y $70 \mathrm{~cm}$ como límite superior de diámetros. Las densidades en área basal resultantes variaron aproximadamente entre 30 y $33 \mathrm{~m}^{2} \mathrm{ha}^{-1}$.

- Lognormal (LOG). Se establecieron diámetros aleatorios con distribución lognormal mediante la función rlnorm() con una media de $3\left(\mathrm{e}^{3}=20,1 \mathrm{~cm}\right)$ y un desvío estándar de $0,5\left(\mathrm{e}^{0,5}=1,8 \mathrm{~cm}\right)$. La distribución fue truncada en $5 \mathrm{~cm}$ como límite inferior y $70 \mathrm{~cm}$ como límite superior de diámetros. Esta distribución produjo valores de área basal similares a los de la distribución normal.

- Bimodal (BIM). Se generó mediante la superposición de dos distribuciones: (1) lognormal con media 2,8 ( $\mathrm{e}^{2,8}$ $=16,4 \mathrm{~cm})$ y un desvío estándar de $0,3\left(\mathrm{e}^{0,3}=1,4 \mathrm{~cm}\right)$ y (2) normal con media $30 \mathrm{~cm}$ y un desvío estándar de $5 \mathrm{~cm}$.

De cada una de las siete situaciones se realizaron 1.000 simulaciones estableciéndose en cada caso 15 puntos al azar con un espaciamiento mínimo de $50 \mathrm{~m}$ mediante la función $\mathrm{rSSi}()$ del paquete "spatstat". En cada punto se contó el número de árboles que presentaban una distancia menor a la establecida como límite dado su diámetro y cada uno de los factores considerados (2, 3, y 4). El espaciamiento de $50 \mathrm{~m}$ entre puntos de conteo evitó la superposición de árboles incluidos ya que la distancia límite para los árboles de mayor diámetro $(70 \mathrm{~cm})$ en el factor más bajo (2) fue de 24,75 m. La decisión de simular un muestreo de sólo 15 puntos tuvo la finalidad de evitar que un muestreo demasiado intenso, para el área simulada, dificultara la manifestación de las tendencias que se pretendía evaluar. En cada simulación se calculó: el área basal media de la población $(\mathrm{AB})$ y la media de la densidad estimada por cada factor. Para el total de las 1.000 simulaciones se calculó el desvío absoluto medio:

$$
D A M=\sum_{1}^{1000} \mid \text { ABbit. }- \text { ABpob. } \mid
$$

Donde, ABbit.: media estimada por recuento angular y $\mathrm{AB}$ pob.: área basal promedio por hectárea del total del área simulada.

Además se calculó el sesgo (S) como la sumatoria desde 1 hasta 1.000 de la diferencia entre la media estimada por cada factor y la calculada para el total del área simulada, con su signo. 

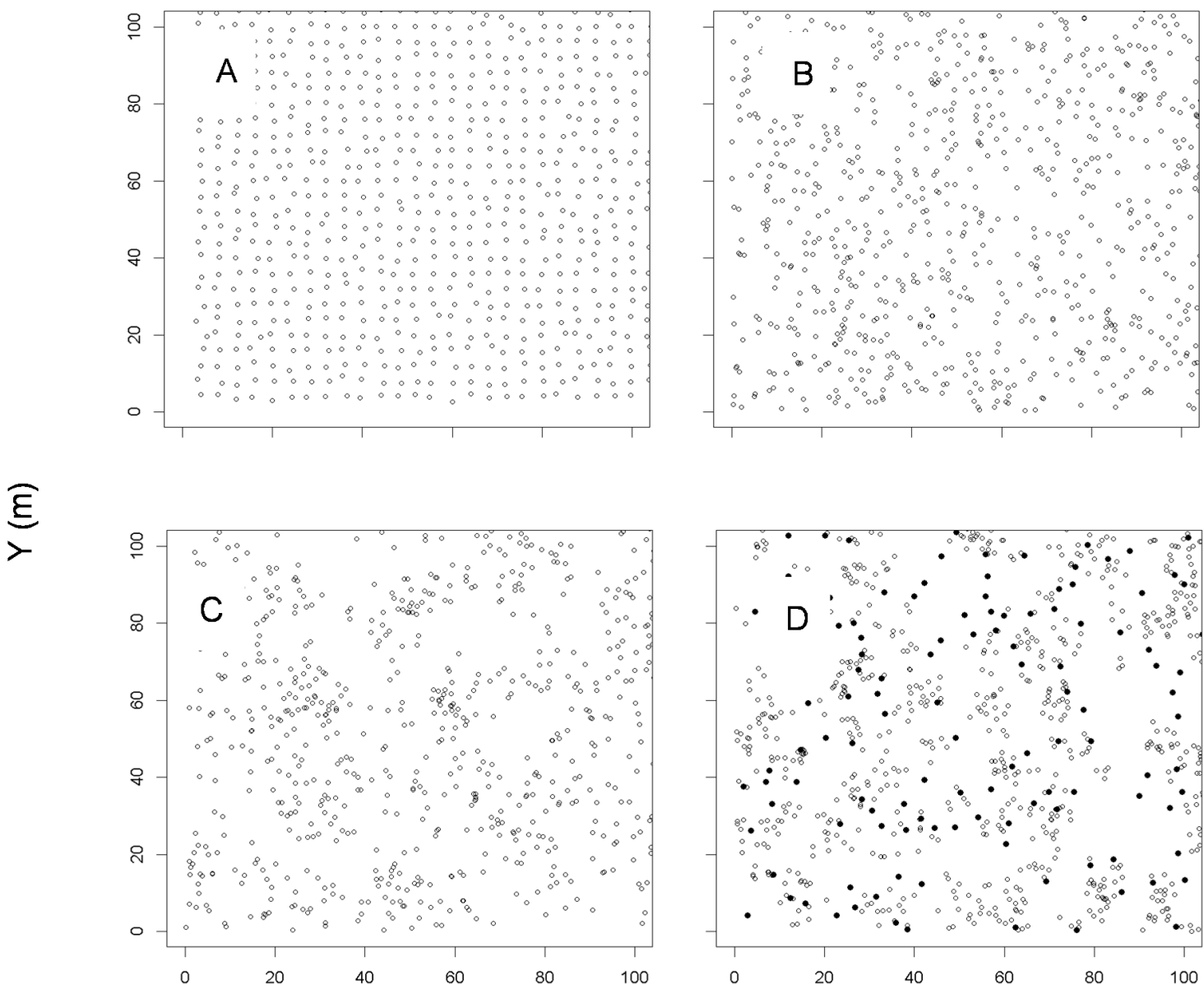

$X(m)$

Figura 2. Distribuciones espaciales de árboles simuladas. A: regular, B: aleatoria, C: agrupada, D: mixta. En la distribución mixta los círculos abiertos representan árboles pequeños y los círculos cerrados representan árboles grandes. Sólo se muestra 1 ha pero las simulaciones fueron de 16 ha.

Simulated tree spatial patterns: A: regular, B: random, C: clumped, D: mixed. In the mixed pattern white circles indicate small trees and black circles indicate large trees. Only 1 ha is shown though 4 ha were simulated.

\section{RESULTADOS}

Las estimaciones del área basal mediante el recuento angular fueron siempre muy cercanas al valor poblacional al considerar el promedio del total de simulaciones (cuadro 1). El sesgo fue siempre inferior a $0,10 \mathrm{~m}^{2} \mathrm{ha}^{-1} \mathrm{y}$ el intervalo de confianza al $95 \%$ contuvo el valor 0 en todos los, casos indicando que no se diferenciaron significativamente de ese valor. En AGR-LOG y MIX-BIN el sesgo presentó una débil tendencia negativa encontrándose entre $-0,15$ y $-0,25 \mathrm{~m}^{2} \mathrm{ha}^{-1}$. El desvío absoluto medio presentó sus valores más bajos en la distribución regular variando, aproximadamente, entre $3 \%$ y $7 \%$ del área basal estimada, mientras que en la distribución aleatoria varió entre $5 \%$ y $8 \%$. Los desvíos absolutos medios más altos se observaron en las distribuciones agrupada (entre $7 \%$ y $10 \%$ ) y mixta (entre $8 \%$ y $11 \%)$. Su relación con la distribución diamétrica fue diferente según la distribución espacial considerada. El desvío medio tendió a ser menor en REG-NOR que en REG-LOG, similar entre AZA-NOR y AZA-LOG, y mayor en AGR-NOR que en AGR-LOG.

\section{DISCUSIÓN}

En todas las situaciones simuladas las estimaciones de la densidad en área basal obtenidas por recuento angular resultaron muy cercanas al valor real. Los valores de sesgo hallados no se diferenciaron de cero y pueden considerarse nulos, en términos prácticos. En las distribuciones espaciales regular y aleatoria no hubo diferencia en el sesgo y la variabilidad de las estimaciones para las distintas distribuciones diamétricas. Esto sugiere que, a pesar de las 


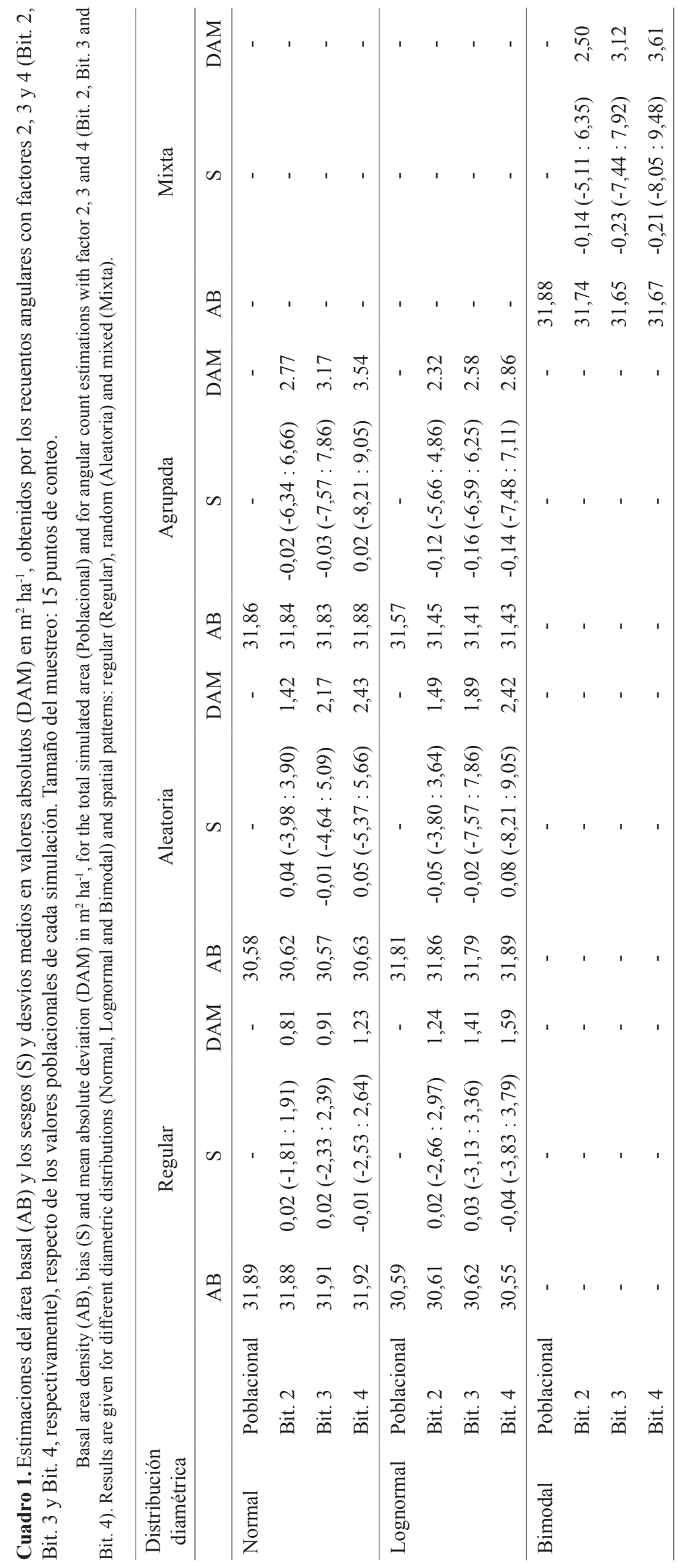


diferencias en las probabilidades de inclusión entre árboles de diferentes diámetros, el aumento de la variabilidad en la distribución diamétrica no introduce sesgo, ni aumenta la variabilidad de las estimaciones por recuento angular, en distribuciones espaciales regulares o aleatorias. En esos casos, aún en un muestreo con un bajo número de puntos, permite obtener una estimación eficiente e insesgada de la densidad en área basal. En las distribuciones espaciales agrupada o mixta (AGR-LOG y MIX-BIM) se observó una débil tendencia a la subestimación de esa variable. Esa tendencia resulta coherente con el resultado esperado por el efecto de las distribuciones espaciales agrupadas sobre los métodos sin parcelas basados en las distancias puntoárbol (Batcheler 1971, Pollard 1971, Engeman et al. 1994, Hijbeek et al. 2013). En distribuciones agrupadas, las estimaciones de la densidad de árboles basadas en la distancia entre puntos de muestreo y el árbol más cercano producen subestimaciones dado que las distancias entre grupos de árboles son mayores que las distancias entre árboles (Batcheler 1971, Hijbeek et al. 2013). Si las distancias entre los puntos de muestreo por recuento angular y los árboles incluidos tienden a ser aumentadas por la distribución espacial agrupada, entonces, la densidad en área basal resulta subestimada. Los árboles pequeños presentan una reducida distancia de inclusión (Kangas y Maltamo 2006, Gregoire y Valentine 2007). En consecuencia, para rodales con similares áreas basales, la subestimación sería mayor en aquel en el cual mayor sea el predominio numérico de árboles pequeños, que por su reducida distancia de inclusión, resultarían más sensibles a ese efecto. Esa podría ser la causa por la que los sesgos tendieron a diferenciarse más de cero en las distribuciones diamétricas lognormal y bimodal en comparación con la distribución normal.

Los resultados de este trabajo indican que el patrón de distribución espacial y la distribución diamétrica pueden afectar la exactitud y precisión de las estimaciones del área basal de un bosque por recuento angular. Sin embargo, dada la baja magnitud de los efectos hallados, estos resultados respaldan, mediante simulaciones en condiciones controladas, un hecho que hasta ahora era conocido solo a través de experiencias prácticas no sistemáticas: el recuento angular de Bitterlich representa un método preciso e insesgado en un amplio rango de patrones espaciales y distribuciones diamétricas.

\section{AGRADECIMIENTOS}

La realización de este trabajo fue financiada por la Facultad de Ciencias Agrarias y Forestales de la Universidad Nacional de La Plata.

\section{REFERENCIAS}

Baddeley A, R Turner. 2005. spatstat: An R Package for Analyzing Spatial Point Patterns. Journal of Statistical Software 12(6):1-42.
Batcheler CL. 1971. Estimation of Density from a Sample of Joint Point and Nearest-Neighbor Distances. Ecology 52(4): 703-709.

Bitterlich W. 1984. The Relascope Idea: relative measurements in forestry. Slough, Inglaterra. Commonwealth Agricultural Bureaux. 242 p.

Burns SL, J F Goya, M F Arturi, PF Yapura, CA Perez. 2010. Stand dynamics, spatial pattern and site quality in Austrocedrus chilensis forests in Patagonia, Argentina. Forest Systems 22(2): 170-178.

Condit R, PS Ashton, P Baker, P Bunyavejchewin, S Gunatilleke, A Gunatilleke, S Hubbell, R Foster, A Itoh, JV LaFrankie, HS Lee, E Losos, N Manokaran, R Sukumar, T Yamakura. 2000. Spatial patterns in the distribution of tropical tree species. Science 288(5): 1414-1418.

Eastaugh CS, H Hasenauer. 2014. Improved estimates of per-plot basal area from angle count inventories. iForest 7(6): 178-185.

Engeman R, R Sugihara, L Pank, L Dusenberry. 1994. A comparison of plotless density estimators using Montecarlo simulation. Ecology 75(6): 1769-1779.

Getzin S, C Dean, F He, JA Trofymow, K Wiegand, T Wiegand. 2006. Spatial patterns and competition of tree species in a Douglas-fir chronosequence on Vancouver Island. Ecography 29(2): 671-682.

Gregoire TG, HT Valentine. 2007? Sampling Strategies for Natural Resources and Environment. Londres, Inglaterra. Chapman-Hall. 492 p.

Hijbeek R, N Koedam, MNI Khan, JG Kairo, J Schoukens, F Dahdouh-Guebas. 2013. An evaluation of plotless sampling using vegetation simulations and field data from a mangrove forest. PLOS ONE 8. Consultado 1 abr. 2015. Disponible en: http://www.ncbi.nlm.nih.gov/pmc/articles/ PMC3695089/pdf/pone.0067201.pdf

Kangas A, M Maltamo, 2006. Forest inventory. Methodology and applications. Berlin, Alemania. Springer. 362 p.

Li L, Z Huang, W Ye, H Cao, S Wei, Z Wang, J Lian, I Sun, K Ma, F He. 2009. Spatial distributions of tree species in a subtropical forest of China. Oikos 118(4): 495-502.

Palley MN, LG Horwitz. 1961. Properties of some random and systematic point sampling estimators. Forest Science 7(1): 52-65.

Podlaski R, M Zasada. 2008. Comparison of selected statistical distributions for modelling the diameter distributions in near-natural Abies-Fagus forests in the Świętokrzyski National Park (Poland). European Journal of Forest Research 127(2):455-463.

Pollard, JH 1971. On distance estimators of density in randomly distributed forests. Biometrics 27(4):991-1002.

R Core Team. 2012. R: A language and environment for statistical computing. R Foundation for Statistical Computing, Vienna, Austria. Consultado 10 feb. 2015. Disponible en http://www.R-project.org/

Salas CE, Reyes M, B Bassaber. 2005. Medición de diámetros fustales con relascopio y forcípula finlandesa: efectos en la estimación de volumen. Bosque 26(2):81-90.

Sheykholeslami A, K Pasha, A Lashaki. 2011. A Study of Tree Distribution in Diameter Classes in Natural Forests of Iran (Case Study: Liresara Forest). Annals of Biological Research 2(5):283-290.

Ritter T, BA Nothdurft, J Saborowski. 2013. Correcting the nondetection bias of angle count sampling. Canadian Journal 
of Forest Research 43(4):344-354.

Sukwong S, W Frayer, E Mogren, Edwin. 1971. Generalized Comparisons of the Precision Fixed-Radius and VariableRadius Plots for Basal-Area Estimates. Forest Science
17(2):263-271.

Whyte AGD, RB Tennent. 1975. Improving estimates of stand basal area in working plan inventories. New Zealand Journal of Forestry 20(1):134-147.

Recibido: 11.05 .15

Aceptado: 08.04.16 
